PROCEEDINGS OF THE

AMERICAN MATHEMATICAL SOCIETY

Volume 135, Number 12, December 2007, Pages 3789-3794

S 0002-9939(07)08970-8

Article electronically published on September 7, 2007

\title{
ON QUASI-ISOMETRIC EMBEDDINGS OF LAMPLIGHTER GROUPS
}

\author{
S. P. INAMDAR AND ANIRUDDHA C. NAOLEKAR
}

(Communicated by Alexander N. Dranishnikov)

\begin{abstract}
We denote by $\Gamma_{G}$ the Lamplighter group of a finite group $G$. In this article, we show that if $G$ and $H$ are two finite groups with at least two elements, then there exists a quasi-isometric embedding from $\Gamma_{G}$ to $\Gamma_{H}$. We also prove that the quasi-isometry group $\mathcal{Q} I\left(\Gamma_{G}\right)$ of $\Gamma_{G}$ contains all finite groups. We then show that the group of automorphisms of $\Gamma_{\mathbb{Z}_{n}}$ has infinite index in $\mathcal{Q} I\left(\Gamma_{\mathbb{Z}_{n}}\right)$.
\end{abstract}

\section{INTRODUCTION}

Recall that if $(X, d)$ and $\left(Y, d^{\prime}\right)$ are metric spaces, then a map $f: X \longrightarrow Y$ is called a $(\lambda, \varepsilon)$ quasi-isometric embedding if there exist constants $\lambda, \varepsilon \geq 0$ such that

$$
\frac{1}{\lambda} d(x, y)-\varepsilon \leq d^{\prime}(f(x), f(y)) \leq \lambda d(x, y)+\varepsilon .
$$

A quasi-isometric embedding $f$ is called a quasi-isometry if there exists a constant $C \geq 0$ such that $d^{\prime}(y, f(X)) \leq C$ for all $y \in Y$. Associated to any metric space $(X, d)$ is its quasi-isometry group $\mathcal{Q} I(X)$. This is the group of all self quasiisometries of $X$ modulo those which are at a bounded distance from the identity.

If $\Gamma$ is a finitely generated group with a finite generating set $\mathcal{A}$, then the word metric corresponding to $\mathcal{A}$ is denoted by $d_{\mathcal{A}}$. If $\mathcal{B}$ is another finite generating set for $\Gamma$, then the metric spaces $\left(\Gamma, d_{\mathcal{A}}\right)$ and $\left(\Gamma, d_{\mathcal{B}}\right)$ are quasi-isometric. We can therefore unambiguously talk about two finitely generated groups being quasi-isometric without referring to the word metrics. In geometric group theory, one studies the properties of finitely generated groups which are invariant under quasi-isometries.

Let $G$ be a finite group. A Lamplighter group $\Gamma_{G}$ is the wreath product of $G$ and $\mathbb{Z}$. Hence, $\Gamma_{G}=\left(\bigoplus_{i \in \mathbb{Z}} G\right) \rtimes \mathbb{Z}$. These groups provide examples to the fact that the property of being virtually solvable and also the property of being virutually torsion free are not geometric properties, that is, they are not preserved under quasi-isometries (4]). From a probabilistic point of view, random walks on the Cayley graph of a Lamplighter group have been extensively studied in [5], 6], 8]. From the point of view of geometric group theory, one is interested in the quasi-isometry classification of the Lamplighter groups. It is known that if $G$ and $F$ are two finite groups such that $\operatorname{ord}\left(G^{k}\right)=\operatorname{ord}\left(F^{l}\right)$ for some positive integers $k, l$,

Received by the editors May 11, 2006 and, in revised form, September 12, 2006 and September 21, 2006.

2000 Mathematics Subject Classification. Primary 20F65; Secondary 20F28.

Key words and phrases. Lamplighter groups, geometric group theory.

(C)2007 American Mathematical Society Reverts to public domain 28 years from publication 
then the groups $\Gamma_{G}$ and $\Gamma_{F}$ are quasi-isometric (see [3, [4]). However, not much is known when the orders of the groups do not satisfy the above condition. In fact, it is not known whether the groups $\Gamma_{\mathbb{Z}_{2}}$ and $\Gamma_{\mathbb{Z}_{3}}$ are quasi-isometric.

We denote by $\Gamma_{n}$ the Lamplighter group $\Gamma_{G}$ of a finite group $G$ of order $n$. In this article, we prove that for any integers $n, m>1$, there exists a quasi-isometric embedding from $\Gamma_{n}$ to $\Gamma_{m}$. We also study $\mathcal{Q} I\left(\Gamma_{n}\right)$, the quasi-isometry group of $\Gamma_{n}$, and prove that it contains all finite groups. As a consequence of this result we see that finite subgroups of $\mathcal{Q} I\left(\Gamma_{n}\right)$ will not be of any help in distinguishing the quasi-isometry classes amongst the Lamplighter groups. On the other hand, we also show that the order of an automorphism of $\Gamma_{\mathbb{Z}_{n}}$ is either infinite or divides $2 n \phi(n)$, where $\phi(n)$ denotes the number of integers which are less than $n$ and are co-prime to $n$. These two results allow us to show that the group of automorphisms of $\Gamma_{\mathbb{Z}_{n}}$ has infinite index in $\mathcal{Q} I\left(\Gamma_{n}\right)$.

\section{Geometry of $\Gamma_{n}$}

We now describe the word metric on $\Gamma_{n}$. We denote by $G_{m}$ the ' $m$ th copy' of $G$ and by $a_{m}$ a typical element of $G_{m}$. Thus, when multiple $a_{i}$ occur in an expression, it is not assumed that all instances refer to the same element of $G_{i}$. Consider the following generating set:

$$
\mathcal{A}=\left\{a_{0}, t, t^{-1} \mid a_{0} \neq e\right\}
$$

of $\Gamma_{n}$. For an integer $k>0$, the subgroup $H_{k}$ of $\Gamma_{n}$ generated by $\left\{a_{0}, t^{k}, t^{-k} \mid a_{0} \neq\right.$ e\} has index $k$ in $\Gamma_{n}$. As $H_{k}$ is isomorphic to $\Gamma_{n^{k}}$, we see that $\Gamma_{n}$ and $\Gamma_{n^{k}}$ are quasi-isometric.

A possible normal form of a word $\omega \in \Gamma_{n}$ is given by $\omega=a_{i_{1}} a_{i_{2}} \cdots a_{i_{r}} t^{m}$ with $i_{1}<i_{2}<\cdots<i_{r}$. If $\omega$ has the above form, then $\omega$ can also be written as

$$
\begin{aligned}
\omega & =\left(t^{i_{1}} a_{0} t^{-i_{1}}\right)\left(t^{i_{2}} a_{0} t^{-i_{2}}\right) \cdots\left(t^{i_{r}} a_{0} t^{-i_{r}}\right) t^{m} \\
& =t^{i_{1}} a_{0} t^{i_{2}-i_{1}} a_{0} t^{i_{3}-i_{2}} \cdots a_{0} t^{i_{r}-i_{r-1}} a_{0} t^{m-i_{r}} .
\end{aligned}
$$

Thus

$$
\ell(\omega) \leq\left|i_{1}\right|+r+\left(i_{r}-i_{1}\right)+\left|m-i_{r}\right| .
$$

On the other hand, $\omega$ can also be written as $\omega=a_{i_{r}} a_{i_{r-1}} \cdots a_{i_{1}} t^{m}$ so that

$$
\omega=t^{i_{r}} a_{0} t^{i_{r-1}-i_{r}} \cdots a_{0} t^{i_{1}-i_{2}} a_{0} t^{m-i_{1}}
$$

and hence

$$
\ell(\omega) \leq\left|i_{r}\right|+r+\left(i_{r}-i_{1}\right)+\left|m-i_{1}\right| .
$$

We shall show that the length $\ell(\omega)$ of $\omega$ is given by the following formula.

Lemma 2.1. Let $\omega \in \Gamma_{n}$ be as above; then

$$
\ell(\omega)=r+\left(i_{r}-i_{1}\right)+\min \left\{\left|i_{1}\right|+\left|m-i_{r}\right|,\left|i_{r}\right|+\left|m-i_{1}\right|\right\} .
$$

Proof. Let $\omega=a_{i_{1}} a_{i_{2}} \cdots a_{i_{r}} t^{m}$ with $i_{1}<i_{2}<\cdots<i_{r}$. Inequalities (2.1) and (2.2) imply that

$$
\ell(\omega) \leq r+\left(i_{r}-i_{1}\right)+\min \left\{\left|i_{1}\right|+\left|m-i_{r}\right|,\left|i_{r}\right|+\left|m-i_{1}\right|\right\} .
$$

Let $P=t^{k_{0}} a_{0} t^{k_{1}} a_{0} \cdots t^{k_{\ell}} a_{0} t^{k_{\ell+1}}$ be any path from 1 to $\omega$ in the Cayley graph. Here $k_{i} \neq 0$ if $0<i<\ell+1$.

First observe that $a_{0}$ occurs at least $r$ times in the path $P$ so that $\ell+1 \geq r$. Next, consider the sequence of the partial sums $k_{0}, k_{0}+k_{1}, \ldots, k_{0}+k_{1}+\cdots+k_{\ell}$ of 
the exponents of $t$ in the path $P$. The indices $i_{1}$ and $i_{r}$ must appear as one of the terms of this sequence. Suppose that $i_{1}$ appears before $i_{r}$ in this sequence at the $j$ th place. Then, $k_{0}+\cdots+k_{j}=i_{1}$. If $a_{i_{r}}$ occurs at the $s$ th place in $P$ with $j<s$, we get $k_{j+1}+\cdots+k_{s}=i_{r}-i_{1}$. Finally, we must have $k_{s+1}+\cdots+k_{\ell}=m-i_{r}$ to achieve the exponent $m$ of $t$. Thus, we see that in this case the length $\ell(P)$ of the path $P$ satisfies the bound

$$
\ell(P)=(\ell+1)+\sum_{0}^{\ell+1}\left|k_{i}\right| \geq r+\left|i_{1}\right|+\left(i_{r}-i_{1}\right)+\left|m-i_{r}\right| .
$$

On the other hand, if $i_{r}$ appears before $i_{1}$ in the sequence of partial sums, similar arguments show that

$$
\ell(P) \geq r+\left|i_{r}\right|+\left(i_{r}-i_{1}\right)+\left|m-i_{1}\right| .
$$

Inequalities (2.4) and (2.5) show that

$$
\ell(\omega) \geq r+\left(i_{r}-i_{1}\right)+\min \left\{\left|i_{1}\right|+\left|m-i_{r}\right|,\left|i_{r}\right|+\left|m-i_{1}\right|\right\} .
$$

This completes the proof of the lemma.

Remark 2.2. By Lemma 2.1, the length of the word $a_{0} \cdots a_{r}$ is $3 r+1$. Thus, in $\Gamma_{n}$, the ball of radius $3 r+1$ around the identity will have at least $(n-1)^{r+1}$ distinct elements. This proves that $\Gamma_{n}$ has exponential growth for all $n>2$. Since $\Gamma_{2}$ contains $\Gamma_{4}$ as a finite index subgroup, $\Gamma_{n}$ has exponential growth for all $n>1$.

Remark 2.3. Another description of the length of a word in the Lamplighter group can be found in [1].

Given two finitely generated groups it is a difficult problem to decide if one quasi-isometrically embeds into the other. Even if there exists a quasi-isometric embedding in one direction there may not be one in the other. Indeed, the free group $F_{2}$ of rank 2 does not quasi-isometrically embed into the infinite cyclic group $\mathbb{Z}$. Using the above description of word length in $\Gamma_{n}$ we show that any two Lamplighter groups can be quasi-isometrically embedded into the each other.

Theorem 2.4. Let $u, v$ be integers greater than 1 . Then there exists a quasiisometric embedding $\Theta_{u, v}: \Gamma_{u} \longrightarrow \Gamma_{v}$.

Proof. We first assume that $1<u \leq v$. Let $\theta: \mathbb{Z}_{u} \rightarrow \mathbb{Z}_{v}$ be a (set theoretic) one-one map with $\theta(e)=e$. Define $\Theta_{u, v}: \Gamma_{u} \rightarrow \Gamma_{v}$ by:

$$
\Theta_{u, v}\left(t^{i} x t^{-i}\right)=t^{i} \theta(x) t^{-i} \text { and } \Theta_{u, v}\left(a_{i_{1}} \cdots a_{i_{r}} t^{m}\right)=\Theta_{u, v}\left(a_{i_{1}}\right) \cdots \Theta_{u, v}\left(a_{i_{r}}\right) t^{m}
$$

where $x$ denotes a non-identity element of $\mathbb{Z}_{u}$.

If $\omega, \tau$ are two elements of $\Gamma_{u}$ with

$$
\begin{aligned}
\omega & =a_{i_{1}} \cdots a_{i_{r}} t^{m}, \\
\tau & =a_{j_{1}} \cdots a_{j_{s}} t^{n},
\end{aligned}
$$

then,

$$
\Theta_{u, v}\left(\omega^{-1} \tau\right)=t^{-m}\left[\Theta_{u, v}\left(a_{i_{1}}\right)\right]^{-1} \cdot s\left[\Theta_{u, v}\left(a_{i_{r}}\right)\right]^{-1} \Theta_{u, v}\left(a_{j_{1}}\right) \cdots \Theta_{u, v}\left(a_{j_{s}}\right) t^{n} .
$$

Since $\Theta_{u, v}$ takes the $i$ th copy of $\mathbb{Z}_{u}$ in $\Gamma_{u}$ to the $i$ th copy of $\mathbb{Z}_{v}$ in $\Gamma_{v}$, the indices that get cancelled (or clubbed together) in the expression of $\omega^{-1} \tau$ are the same as the indices that get cancelled (or clubbed together) in $\Theta_{u, v}(\omega)^{-1} \Theta_{u, v}(\tau)$, and hence $\ell\left(\omega^{-1} \tau\right)=\ell\left(\Theta_{u, v}(\omega)^{-1} \Theta_{u, v}(\tau)\right)$. Therefore, $\Theta_{u, v}$ is an isometry. 
In case $u>v$, we choose a positive integer $k$ such that $u<v^{k}$ and construct $\Theta_{u, v^{k}}: \Gamma_{u} \longrightarrow \Gamma_{v^{k}}$ as above. As observed before, there exists a quasi-isometry $\chi: \Gamma_{v^{k}} \longrightarrow \Gamma_{v}$. Then $\chi \circ \Theta_{u, v^{k}}: \Gamma_{u} \longrightarrow \Gamma_{v}$ is a quasi-isometric embedding. This completes the proof.

Remark 2.5. As $\Gamma_{1}=\mathbb{Z}$, there exists a quasi-isometric embedding of $\Gamma_{1}$ inside $\Gamma_{v}$, for all positive integers $v$. On the other hand, for all $v>1$, Remark 2.2 shows that $\Gamma_{v}$ has exponential growth so that there does not exist any quasi-isometric embedding from $\Gamma_{v}$ to $\Gamma_{1}$.

\section{QuASI-ISOMETRY GROUP OF $\Gamma_{n}$}

Let $\Gamma$ and $\Gamma^{\prime}$ be two finitely generated groups. If $\varphi: \Gamma \longrightarrow \Gamma^{\prime}$ is a group homomorphism, then $\varphi$ is a quasi-isometry if and only if both its kernel and cokernel are finite. Thus, for any finitely generated group $\Gamma$, we have a canonical homomorphism $\theta: \operatorname{Aut}(\Gamma) \longrightarrow \mathcal{Q} I(\Gamma)$. We shall denote by $C(g)$ the centralizer of $g$ in $\Gamma$. The virtual center $K(\Gamma)$ of $\Gamma$ is the group

$$
K(\Gamma)=\{g \in \Gamma \mid[\Gamma: C(g)]<\infty\} .
$$

In [7, it was proved that the canonical homomorphism $\theta: \operatorname{Aut}(\Gamma) \longrightarrow \mathcal{Q} I(\Gamma)$ is injective if $K(\Gamma)=0$.

The quasi-isometry groups of $\Gamma_{n}$ are not known. Their structure has been conjectured in [9]. In this section we show that, for any $n>1$, the quasi-isometry group $\mathcal{Q} I\left(\Gamma_{n}\right)$ contains all finite groups. Thus, the torsion elements in the groups $\mathcal{Q} I\left(\Gamma_{n}\right)$ cannot be used to distinguish the quasi-isometry classes amongst the Lamplighter groups. We begin with the following.

Lemma 3.1. Let $G$ be a finite group with $|G|>1$. Then $K\left(\Gamma_{G}\right)=0$. Consequently, Aut $\left(\Gamma_{G}\right)$ embeds into $\mathcal{Q} I\left(\Gamma_{G}\right)$.

Proof. Note that the virtual center consists of precisely those elements having finitely many conjugates. Since,

$$
t^{i}\left(a_{i_{1}} \cdots a_{i_{r}} t^{m}\right) t^{-i}=a_{i_{1}+i} \cdots a_{i_{r}+i} t^{m}
$$

and since none of the $a_{i}$ 's commute with $t^{m}$, we see that every non-identity element of $\Gamma_{G}$ has infinitely many conjugates.

Lemma 3.2. Let $G$ be a finite group. Let $\theta: G \longrightarrow G$ be an automorphism. Then $\theta$ extends to an automorphism $\Theta$ of $\Gamma_{G}$.

Proof. We define $\Theta$ by $\Theta\left(a_{i_{1}} \cdots a_{i_{r}} t^{m}\right)=\theta(a)_{i_{1}} \cdots \theta(a)_{i_{r}} t^{m}$.

Remark 3.3. The extension of $\theta$ is not unique. For example, if $G$ is abelian, the identity automorphism of $G$ can be extended as conjugation by any finite order element of $\Gamma_{G}$. Since $t$ does not commute with finite order elements of $\Gamma_{G}$, this extension is not an identity automorphism.

Theorem 3.4. $\mathcal{Q} I\left(\Gamma_{n}\right)$ contains all finite groups.

Proof. Let $k$ be a positive integer. As before, let $H_{k}$ denote the index $k$ subgroup of the Lamplighter group $\Gamma_{n}$. If $C_{k, n}$ denotes the direct sum of $k$ copies of $\mathbb{Z}_{n}$, then $H_{k}=\Gamma_{C_{k, n}}$. By Lemma 3.2, the permutation group $S_{k}$ is a subgroup of $\operatorname{Aut}\left(\Gamma_{C_{k, n}}\right)$. By Lemma 3.1. we have $S_{k} \subset \mathcal{Q} I\left(H_{k}\right)$. However, $\mathcal{Q} I\left(\Gamma_{n}\right)=\mathcal{Q} I\left(H_{k}\right)$ as $H_{k}$ is quasi-isometric to $\Gamma_{n}$. This proves the theorem. 
We contrast the above theorem with the following result.

For a positive integer $n$, let $\phi(n)$ denote the order of the group of units in $\mathbb{Z}_{n}$. Let $G$ be a finite group. The support of an element $w=a_{j_{1}} \cdots a_{j_{s}} \in \Gamma_{G}$ is the set $\left\{j_{1}, \ldots, j_{s}\right\}$, and we denote it by $\operatorname{Supp}(w)$.

Theorem 3.5. Let $\varphi \in A u t\left(\Gamma_{\mathbb{Z}_{n}}\right)$ be an element of finite order. Then the order of $\varphi$ divides $2 n \phi(n)$.

Proof. We denote by $G_{m}$ the ' $m$ th copy' of $\mathbb{Z}_{n}$. A typical element of $G_{m}$ is denoted by $a_{m}$. Let $x_{0}$ denote a generator of $G_{0}$ and let $x_{m}=t^{m} x_{0} t^{-m}$. As $\bigoplus_{m} G_{m}$ is precisely the subgroup of elements of finite order in $\Gamma_{n}$, it is characteristic. Hence every automorphism $\varphi: \Gamma_{n} \longrightarrow \Gamma_{n}$ induces an automorphism $\widetilde{\varphi}$ of $\Gamma_{n} / \bigoplus_{m} G_{m} \cong$ $\mathbb{Z}$. An automorphism $\varphi$ of $\Gamma_{n}$ is said to be even if $\widetilde{\varphi}$ is the identity automorphism and odd otherwise. Since the square of an odd automorphism is even, it is enough to show that the order of an even finite order automorphism divides $n \phi(n)$.

Assume that $\varphi: \Gamma_{n} \longrightarrow \Gamma_{n}$ is an even automorphism. Let $\varphi\left(x_{0}\right)=a_{i_{1}} \cdots a_{i_{r}}$ for some integers $i_{1}<\cdots<i_{r}$. Also, as $\varphi$ is even, we must have $\varphi(t)=w t$ for some finite order element $w \in \Gamma_{n}$.

Define $w_{i}$ by $\varphi\left(t^{i}\right)=w_{i} t^{i}$. Then, $\varphi\left(x_{l}\right)=\varphi\left(t^{l} x_{0} t^{-l}\right)=a_{i_{1}+l} \cdots a_{i_{r}+l}$, for any integer $l$. Thus $\varphi^{2}\left(x_{0}\right)=a_{2 i_{1}} z_{2} a_{2 i_{r}}$, where $z_{2}=a_{j_{1}} \cdots a_{j_{s}}$ with $2 i_{1}<j_{q}<2 i_{r}$ for all $j_{q}$. Inductively, we see that $\varphi^{n}\left(x_{0}\right)=a_{n i_{1}} z_{n} a_{n i_{r}}$, where $z_{n}$ is the product of terms from $G_{i}$ with $n i_{1}<i<n i_{r}$. Therefore, for any even automorphism $\varphi$ of $\Gamma_{n}$ such that $\varphi\left(x_{0}\right)=a_{i_{1}} \cdots a_{i_{r}}$, we must have $\left\{n i_{1}, n i_{r}\right\} \subset \operatorname{Supp}\left(\varphi^{n}\left(x_{0}\right)\right)$.

Therefore, if $\varphi$ is a finite order even automorphism, we must have $\varphi\left(x_{0}\right) \in G_{0}$. Hence any such $\varphi$ induces an automorphism of $G_{0}$. This implies that $\varphi^{\phi(n)}\left(x_{0}\right)=x_{0}$ and $\varphi^{\phi(n)}(t)=w_{\phi(n)} t$. Since the order of $w_{\phi(n)}$ divides $n$, we see that $\varphi^{n \phi(n)} \equiv I d$. This means that the order of $\varphi$ divides $n \phi(n)$.

Theorem 3.6. $\operatorname{Aut}\left(\Gamma_{\mathbb{Z}_{n}}\right)$ has infinite index in $\mathcal{Q} I\left(\Gamma_{n}\right)$.

Proof. For any positive integer $m$, consider the inclusion $S_{2 m} \subset \mathcal{Q} I\left(\Gamma_{n}\right)$ given by Theorem [3.4. For any disjoint $m$-cycles $\sigma, \tau \in S_{2 m}$, the quasi-isometry given by $\sigma^{-1} \tau$ has order $m$. Therefore, by Theorem 3.5, $\sigma^{-1} \tau \notin A u t\left(\Gamma_{\mathbb{Z}_{n}}\right)$ for large $m$. Hence any such pair $\sigma, \tau$ must belong to distinct cosets of $\Gamma_{\mathbb{Z}_{n}}$ in $\mathcal{Q} I\left(\Gamma_{n}\right)$. As the number of such pairwise disjoint $m$-cycles is not bounded in $\mathcal{Q} I\left(\Gamma_{n}\right)$, the theorem follows.

\section{REFERENCES}

1. S. Cleary; J. Taback, Dead end words in lamplighter groups and other wreath products. Q. J. Math. 56 (2005), no. 2, 165-178. MR2143495 (2006h:20055)

2. S. Cleary; J. Taback, Metric properties of the lamplighter group as an automata group. Geometric methods in group theory, Contemp. Math., 372, Amer. Math. Soc., Providence, RI, 2005, pp. 207-218. MR2140090 (2006d:20071)

3. P. de la Harpe, Topics in geometric group theory. Chicago Lectures in Mathematics. University of Chicago Press, Chicago, IL, 2000. vi+310 pp. MR 1786869 (2001i:20081)

4. A. Dyubina, Instability of the virtual solvability and the property of being virtually torsion-free for quasi-isometric groups. Internat. Math. Res. Notices 2000, no. 21, 1097-1101. MR 1800990 (2001j:20060)

5. R. I. Grigorchuk; A. Zuk, The lamplighter group as a group generated by a 2-state automaton, and its spectrum. Geom. Dedicata 87 (2001), no. 1-3, 209-244. MR.1866850(2002j:60009)

6. R. Lyons; R. Pementale, and Y. Peres, Random walks on the lamplighter group, Ann. Probab. 24 (1996), no. 4, 1993-2006 MR1415237(97j:60014) 
7. A. C. Naolekar; P. Sankaran, Bounded automorphisms and quasi-isometries of finitely generated groups. Jour. Group Theory 8 (2005), 515-522. MR2152695 (2006d:20061)

8. D. Revelle, Heat kernel asymptotics on the lamplighter group. Electron. Comm. Probab. 8 (2003), 142-154 MR2042753 (2005e:60100)

9. K. Wortman, A finitely-presented solvable group with a small quasi-isometry group, preprint 2005.

Department of Theoretical Statistics and Mathematics, Indian Statistical Institute, Bangalore Centre, 8Th Mile, Mysore Road, Bangalore, India 560059

E-mail address: inamdar@ns.isibang.ac.in

Department of Theoretical Statistics and Mathematics, Indian Statistical Institute, Bangalore Centre, 8Th Mile, Mysore Road, Bangalore, India 560059

E-mail address: ani@ns.isibang.ac.in 